Ecology, 100(10), 2019, e02794

(C) 2019 by the Ecological Society of America

\section{Not even wrong: Comment by Loreau and Hector}

\author{
Michel Loreau ${ }^{1,3}$ AND ANDY Hector ${ }^{2}$ \\ Citation: Loreau, M., and A. Hector. 2019. Not even \\ wrong: Comment by Loreau and Hector. Ecology 100 \\ (10):e02794. 10.1002/ecy.2794
}

In a new paper entitled "Not even wrong: The spurious measurement of biodiversity's effects on ecosystem functioning," Pillai and Gouhier (2019) question the validity of the additive partition of biodiversity effects that we proposed $18 \mathrm{yr}$ ago (Loreau and Hector 2001) and that has become a classic in the biodiversity and ecosystem functioning $(\mathrm{BEF})$ research field. Here we show that their critique misinterprets the goal of the BEF research program; in effect, they propose another research program that is both fundamentally different from $\mathrm{BEF}$ and logically inconsistent. In particular, they seek to measure biodiversity effects beyond and above those of species coexistence, an impossible task because biodiversity and coexistence are inseparable concepts. Once the direct and indirect effects of species coexistence are fully accounted for, the residual effect of biodiversity that they propose to study should be zero. We conclude that BEF research should rejuvenate and develop in new directions, but this effort will be successful only if it builds upon the foundations laid down by past research.

In the past $25 \mathrm{yr}$ or so remarkable progress has been made toward understanding how biodiversity affects ecosystem functioning and thereby human societies. After an initial phase of controversy, the scientific community working in the new biodiversity and ecosystem functioning (BEF) research field was able to reach consensus on the conceptual foundations of the field and on a wide range of key results and uncertainties (Loreau et al. 2001, Hooper et al. 2005, Cardinale et al. 2012, Isbell et al. 2017). This consensus was made possible not only by a conscious effort to move beyond differences in

Manuscript received 15 February 2019; accepted 10 June 2019. Corresponding Editor: Donald R. Strong.

${ }^{1}$ Centre for Biodiversity Theory and Modelling, Theoretical and Experimental Ecology Station, CNRS, 2 route du CNRS, 09200 Moulis France.

${ }^{2}$ Department of Plant Sciences University of Oxford, Oxford OX1 3RB UK.

${ }^{3}$ E-mail: michel.loreau@sete.cnrs.fr opinion, but also by new theoretical and methodological advances (Loreau 2010b). The methodology that we proposed in 2001 (Loreau and Hector 2001) played a key role in this consensus-building process because it allowed researchers to partition the net effect of biodiversity on ecosystem functioning into two additive components, a selection effect and a complementarity effect, that have different interpretations and implications. The Loreau-Hector (LH) partition has since been used in hundreds of empirical studies and the paper in which we proposed it has become a classic in the field (https://ref lectionsonpaperspast.wordpress.com/2018/02/01/revisitingloreau-and-hector-2001/).

In a new paper entitled "Not even wrong: The spurious measurement of biodiversity's effects on ecosystem functioning," Pillai and Gouhier (2019) question the validity of the LH partition, and thus, indirectly, of all the conclusions that have been drawn so far about the effects of biodiversity on ecosystem functioning. Pillai and Gouhier (PG) claim that our classic partition is based on "flawed mathematics," thereby uncovering "the logical and mathematical flaws at the heart of the BEF research program," and that it "results in spurious measurements of biodiversity effects." At the same time, they admit that the LH partition "is still formally correct." Although it is hard to see how a method can be simultaneously "formally correct" and based on "flawed mathematics," we do not intend to focus here on the logical inconsistencies that pervade PG's critique. Instead, we want to focus on a much more serious issue: the new foundations on which they propose to rebuild $\mathrm{BEF}$ research are conceptually misleading and incompatible with the objectives of the BEF research program.

In fact, the main thrust of PG's critique is not about the $\mathrm{LH}$ partition, but about the BEF research program itself. The formal theoretical framework they present is superfluous to understand the points they make. In essence, $\mathrm{PG}$ are claiming that the biodiversity effects that have been detected and measured in BEF experiments (whether using the LH partition or not) are spurious because they are "an artefact of coexistence." They believe that biodiversity effects on ecosystem functioning should be measured after accounting for the effects of species coexistence. Accordingly, they propose to change the null hypothesis upon which the BEF research program is based: instead of using the classical null hypothesis that species are functionally redundant and hence that changing their diversity does not affect ecosystem functioning, they propose an alternative null hypothesis "that accounts for the positive effects that coexistence in itself is likely to have on aggregate ecosystem properties." (Note that functional redundancy [Loreau 2004] does not mean equivalence, as different species generally have different 
monoculture yields. PG appear to repeatedly confuse the null hypothesis of functional redundancy used in the LH partition and the null hypothesis of equivalence used in Hubbell's [2001] neutral theory.) Thus, they first derive a prediction on ecosystem functioning in the presence of species coexistence, which they use as their null hypothesis, and then they take the difference between the observed level of ecosystem functioning and this prediction as a measure of the effect of biodiversity on ecosystem functioning. Clearly, what PG have in mind is an altogether different research program, which indeed requires a different null hypothesis and a different methodology. What PG do not appear to realize, however, is that this new research program is, first, completely at odds with the BEF research program that they criticize and, second, logically inconsistent.

The BEF research program was designed to answer a simple yet fundamental question: does biodiversity affect ecosystem functioning? Does a species-rich community differ significantly in its functional processes from a species-poor community, the quintessence of which is the monoculture used in modern agriculture? The answer to this basic question was far from trivial when BEF was conceived in the early 1990s, because there was a wide range of theories on species coexistence, several of which explained species diversity by nonequilibrium processes precluding stable coexistence. This was the case, for instance, of the "intermediate disturbance hypothesis" and other hypotheses based on transient, nonequilibrium coexistence (Huston 1979), which were still influential at that time. Another example is neutral theory (Hubbell 2001), which became influential a decade or so later. Niche partitioning and stable coexistence in species-rich communities were so little established at that time that the first wave of debate that stirred the BEF research field during the 1990s and early 2000s was precisely on the issue whether biodiversity effects on ecosystem functioning could be entirely due to selection effects, that is, due to which species came to dominate mixtures in the absence of any niche differentiation (Huston 1997). The LH partition was designed as a methodology to test this hypothesis, not to "infer or ascribe causal effects," as erroneously interpreted by PG -we already discussed this specific issue at length in a previous contribution (Loreau et al. 2012). Thus, when PG brush aside the "null expectations based on neutral or zero-sum game assumptions" based on the argument that "it is now widely recognized that niche partitioning is common in nature," and when they claim that "the presence of a positive relationship in most BEF studies is thus unsurprising and largely trivial," they simply ignore the history of their science. Species coexistence could be seen as the central question in community ecology (Tokeshi 1999); plant and agricultural sciences have used the zero-sum game assumption as their null hypothesis for nearly 70 yr (De Wit 1960, Harper 1977).
Niche partitioning between species or phenotypes, especially in plant communities, is not a trivial phenomenon; its functional consequences are, to a large extent, precisely what $\mathrm{BEF}$ research was designed to explore, test, and understand. BEF theory has always made clear that there is a tight link between species coexistence and the BEF relationship (Loreau 2010a), a foundational idea that can be traced back to Darwin's realization of the ecosystem-level consequences of his "principle of divergence" of species into different ecological niches (Hector and Hooper 2002). This tight link is a strength, not a weakness, because it connects community ecology and ecosystem ecology, which were widely separated until the emergence of the BEF research field (Naeem 2002, Loreau 2010b).

To remove what they see as "trivial" effects of species coexistence, PG use the matrix of pairwise interspecific competition coefficients and derive a baseline expectation supposed to account for coexistence but not diversity (their Eq. 3). In doing so, $\mathrm{PG}$ are proposing a research program that has nothing in common with the BEF research program. At only one point in their paper do they make it clear that what they have in mind is not to test the effects of biodiversity on ecosystem functioning, but instead to test whether "the aggregate ecosystem property of a community is likely determined by higherorder interactions between species, or other previously unaccounted for nonlinear effects." We have nothing against studying higher-order interactions, which are interesting, and already studied, in their own right (Levine et al. 2017). But if this is their intent, PG should be consistent and stop criticizing the BEF research program, which has another goal. Broadly speaking, BEF research seeks to identify and understand all the ways in which biodiversity affects ecosystem functioning, whether these effects are due to species differences (via selection effects), pairwise species interactions, higher-order interactions, or other processes that contribute to complementarity and selection. In this context, the monoculture is the natural baseline, because a single species or type corresponds to the presence of life but the absence of biodiversity. Other baselines, however, are possible if one is interested in detecting the additional effects of biodiversity beyond two or any other number of species (although we already know from theory and experiments that these effects often become gradually weaker as the number of species increases). In contrast, a research program targeted at detecting and quantifying higher-order interactions is much more specific; its natural baseline should indeed be a diverse community in which pairwise interactions are included, but higher-order interactions are excluded. Note that quantifying higher-order interactions differs from the objective of quantifying the additional effects of biodiversity beyond two species, as BEF theory predicts positive BEF relationships beyond two species even in the absence of any higher-order interactions 
(Gross and Cardinale 2005, Loreau 2010a). By failing to distinguish between two research programs with different objectives and baselines, $\mathrm{PG}$ are generating a huge amount of potential confusion.

The confusion conveyed by PG's critique, however, is much deeper than a mere confusion between two research programs. Their confusion is rooted in an erroneous interpretation of the very concepts of coexistence and diversity. PG seek to measure biodiversity effects beyond and above those of species coexistence. But this is conceptually an impossible task because biodiversity and coexistence are inseparable. Biodiversity is nothing other than a manifestation of the coexistence of species or types (Tokeshi 1999) - no coexistence, no diversity. PG seem to confuse coexistence and the stable local coexistence of species pairs, which is a strongly limited subset of the broader coexistence problem. Coexistence can also be transient and/or nonlocal, that is, driven by regional processes (as in neutral or metacommunity theories), and it can hinge on processes involving more than two species (e.g., higher-order and multitrophic interactions). As a result, coexistence theory is multifaceted, and it predicts different $\mathrm{BEF}$ relationships depending on the mechanism that drives coexistence (Loreau 2010a). If PG wanted to be serious about removing the effects of species coexistence, they should not simply remove the effects of pairwise interactions - they should remove all possible effects of species coexistence in multispecies communities. They will then probably end up in the tautological conclusion that biodiversity has no additional effect on ecosystem functioning, because its effects have been removed by removing the effects of coexistence. The only case in which biodiversity effects on ecosystem functioning may be expected to be unrelated to species coexistence is when diversity has indirect feedback effects through other ecosystem components - a case that PG do not consider.

What PG call "the biodiversity effect," $\Delta \phi_{\mathrm{B}}$, is, in fact, the difference between the total effect of coexistence or biodiversity (including all kinds of processes that affect the community) and its partial effect due to local pairwise interactions. But there is no reason to restrict the effect of coexistence to this partial effect. As a matter of fact, pairwise interactions are but one way to approach coexistence, which can even be misleading under some conditions as higher-order interactions can emerge even from the simplest mechanisms of coexistence (Letten and Stouffer 2019). Thus, the fact that $\Delta \phi_{\mathrm{B}}$ is nonzero may simply reveal that not all mechanisms of coexistence have been taken into account. Again, we are not implying that computing this difference is uninteresting-it can be interesting to detect additional effects of coexistence or biodiversity that are not captured by local pairwise interactions. However, this is not what BEF theory, experiments, and methodology have sought to predict and test and it is unfair and misleading to criticize them on this basis. Claiming that the LH partition and the
$\mathrm{BEF}$ research program are flawed because they have done exactly what they aimed to do is deeply illogical.

The results of PG's analysis also seem to contradict their own approach. Because their so-called "biodiversity effect,", $\Delta \phi_{\mathrm{B}}$, is expected to be zero when all mechanisms of coexistence are considered, how then can it possibly be nonzero in their numerical simulations (their Fig. 3)? Although, amazingly enough, neither the main text nor the supplementary material of their paper provides any information on the model they used to perform these simulations, it is not difficult to see that something must be wrong with either their results or their model. Their linear equation (3) is strictly valid only for a classical Lotka-Volterra model in which species' per-capita population growth rates are linear functions of population densities or biomasses. By construction, this linearity allows only pairwise interactions and precludes any type of higher-order interactions. Therefore, either PG used a Lotka-Volterra model and their results are erroneous, since $\Delta \phi_{\mathrm{B}}$ must then be zero-unless some species went extinct and these extinctions were ignored-or they used another model with strong nonlinearities and/or higher-order interactions and their Eq. 3 does not apply. In either case, their approach and conclusions cannot hold. Once species coexistence is fully accounted for, the residual effect of biodiversity should be zero, because biodiversity and coexistence are one and the same thing in the absence of ecosystem-level feedbacks.

The second part of PG's paper about the nonlinearity issue is more technical and less fundamental; therefore, we only discuss it briefly in this short comment. PG basically argue that the response of ecosystem functioning to a change in the abundance of each species is concave, thereby yielding a nonlinearity that artificially inflates biodiversity effects on ecosystem functioning in a baseline neutral community. This is incorrect, for two reasons. First, biodiversity experiments typically use a substitutive design in which total abundance is kept constant. Second, species are equivalent by definition in a neutral community, so that ecosystem functioning should respond nonlinearly to their total abundance, not to the abundance of each species separately. Therefore, ecosystem functioning is expected to remain constant irrespective of variations in biodiversity in neutral communities. There are other more general issues of nonlinearity, which were not raised by PG, that deserve further consideration. We trust that all ecologists will know that linear models are used as first-order approximations of a more complex, nonlinear reality. The LH partition is linear, in the sense that it quantifies biodiversity effects based on a linear relationship between species' deviations from the null expectation and their monoculture yields, and we have never claimed that it would provide an accurate estimate of biodiversity effects under strong nonlinearities in this relationship. Recent developments 
in BEF research precisely address these more general nonlinearity issues. For instance, Baert et al. (2017) presented a nonlinear extension of the LH partition; Connolly et al. (2013) proposed a nonlinear diversityinteractions model; and Jaillard et al. (2018) developed a novel approach that does not require any linearity or nonlinearity assumption to disentangle the effects of species composition and species interactions on ecosystem functioning. All of these new approaches confirmed the significant effects of biodiversity on ecosystem functioning that were found in previous analyses, which suggests that, although nonlinearity does exist, it is not a critical issue that affects the conclusions reached by the LH partition qualitatively and systematically. PG ignore these recent developments altogether.

$\mathrm{BEF}$ research emerged some $25 \mathrm{yr}$ ago, and during these $25 \mathrm{yr}$ it grew particularly rapidly into a nowmature research field. It is probably time for $\mathrm{BEF}$ to rejuvenate and develop new research directions. New exciting challenges include disentangling the mechanisms underlying biodiversity effects on ecosystem functioning to build predictive models, applying the principles established by theory and experiments to the provision of ecosystem services in natural and managed ecosystems under realistic environmental change scenarios, scaling up biodiversity effects to large temporal and spatial scales, and closing the feedback loop between biodiversity and people (Cardinale et al. 2012, Isbell et al. 2017). But future research should build on the major findings of the past quarter century - that biodiversity impacts ecosystem functioning precisely because the same interspecific differences that allow species to coexist also lead to greater ecosystem productivity and stability at higher diversity. It is inappropriate and counterproductive to redefine terms, as have PG, in a way that ignores the findings of hundreds of experiments and the logic of relevant theory.

\section{ACKNOWLEDGMENTS}

We thank Jean-François Arnoldi, Matthieu Barbier, Adam Clark, Claire de Mazancourt, Forest Isbell, Jose Montoya, Bernhard Schmid and Dave Tilman for valuable comments on the manuscript. ML was supported by the TULIP Laboratory of Excellence (ANR-10-LABX-41) and by the BIOSTASES Advanced Grant, funded by the European Research Council under the European Union's Horizon 2020 research and innovation program (grant agreement No 666971).

\section{Literature Cited}

Baert, J. M., S. Jaspers, C. R. Janssen, F. D. Laender, and M. Aerts. 2017. Nonlinear partitioning of biodiversity effects on ecosystem functioning. Methods in Ecology and Evolution $8: 1233-1240$.
Cardinale, B. J. et al. 2012. Biodiversity loss and its impact on humanity. Nature 486:59-67.

Connolly, J. et al. 2013. An improved model to predict the effects of changing biodiversity levels on ecosystem function. Journal of Ecology 101:344-355.

De Wit, C. T. 1960. On competition. Verslag Landbouwkundig Onderzoek 66:1-82.

Gross, K., and B. J. Cardinale. 2005. The functional consequences of random vs. ordered species extinctions. Ecology Letters 8:409-418.

Harper, J. L. 1977. Plant population biology. Academic Press, London, UK.

Hector, A., and R. Hooper. 2002. Darwin and the first ecological experiment. Science 295:639-640.

Hooper, D. U. et al. 2005. Effects of biodiversity on ecosystem functioning: A consensus of current knowledge. Ecological Monographs 75:3-35.

Hubbell, S. P. 2001. The unified neutral theory of biodiversity and biogeography. Princeton University Press, Princeton, New Jersey, USA.

Huston, M. A. 1979. A general hypothesis of species diversity. American Naturalist 113:81-101.

Huston, M. A. 1997. Hidden treatments in ecological experiments: Re-evaluating the ecosystem function of biodiversity. Oecologia 110:449-460.

Isbell, F. et al. 2017. Linking the influence and dependence of people on biodiversity across scales. Nature 546:65-72.

Jaillard, B., C. Richon, P. Deleporte, M. Loreau, and C. Violle. 2018. An a posteriori species clustering for quantifying the effects of species interactions on ecosystem functioning. Methods in Ecology and Evolution 9:704-715.

Letten, A. D., and D. B. Stouffer. 2019. The mechanistic basis for higher-order interactions and non-additivity in competitive communities. Ecology Letters 22:423-436.

Levine, J. M., J. Bascompte, P. B. Adler, and S. Allesina. 2017. Beyond pairwise mechanisms of species coexistence in complex communities. Nature 546:56-64.

Loreau, M. 2004. Does functional redundancy exist? Oikos 104:606-611.

Loreau, M. 2010a. From populations to ecosystems: Theoretical foundations for a new ecological synthesis. Princeton University Press, Princeton, New Jersey, USA.

Loreau, M. 2010b. Linking biodiversity and ecosystems: Towards a unifying ecological theory. Philosophical Transactions of the Royal Society B 365:49-60.

Loreau, M. et al. 2001. Biodiversity and ecosystem functioning: Current knowledge and future challenges. Science 294:804 808.

Loreau, M., and A. Hector. 2001. Partitioning selection and complementarity in biodiversity experiments. Nature 412:72-76.

Loreau, M., J. Sapijanskas, F. Isbell, and A. Hector. 2012. Niche and fitness differences relate the maintenance of diversity to ecosystem function: Comment. Ecology 93:1482-1487.

Naeem, S. 2002. Ecosystem consequences of biodiversity loss: The evolution of a paradigm. Ecology 83:1537-1552.

Pillai, P., and T. C. Gouhier. 2019. Not even wrong: The spurious measurement of biodiversity's effects on ecosystem functioning. Ecology. https://doi.org/10.1002/ecy.2645.

Tokeshi, M. 1999. Species coexistence: Ecological and evolutionary perspectives. Blackwell Science, Malden, Massachusetts, USA. 